\title{
Ozone layer chemists represent Nobel 'first'
}

London. The Nobel prizes have often been criticized for failing to recognize the contributions of Earth and environmental scientists. That complaint may be heard less often following last week's awards of the chemistry prize to Paul Crutzen of the MaxPlanck-Institute for Chemistry in Mainz, Germany, Mario Molina of the Massachusetts Institute of Technology and F. Sherwood Rowland of the University of California at Irvine, for their work in atmospheric chemistry.

The award citation highlights in particular their role in identifying the threat of the depletion of stratospheric ozone by anthropogenic compounds, and in elucidating the mechanisms by which this occurs.

Ozone is a trace constituent of the atmosphere, formed through photolysis of molecular oxygen Chemistry laureates: Crutzen (left), Rowland (centre) and Molina by the ultraviolet component of (right) helped to identify the threat of stratospheric ozone depletion. sunlight. The highest concentra-

tions are found in the stratosphere at an altitude of between 15 and $40 \mathrm{~km}$; this 'ozone layer' absorbs most solar ultraviolet radiation. Identified in the early part of the century, the ozone layer was recognized in the 1930 s to be a dynamic phenomenon, as ozone is constantly consumed and regenerated by natural processes.

Crutzen showed in 1970 that these processes are coupled to the biogeochemical cycling of trace gases at ground level. He demonstrated that the nitrogen oxides $\mathrm{NO}$ and $\mathrm{NO}_{2}$, formed largely from nitrous oxide $\left(\mathrm{N}_{2} \mathrm{O}\right)$ emitted by biota, catalyse the destruction of ozone. Ralph Cicerone of the University of California at Irvine says that this work "first brought ozone to the fore", showing that it could be affected by human activities. The following year, Harold Johnston suggested that nitrogen oxides released from supersonic aircraft might destroy stratospheric ozone.

More widespread concern followed the suggestion by Rowland and Molina in 1974 that chlorofluorocarbons (CFCs) used industrially as aerosol propellants, foamblowing agents and refrigerator coolants might decompose in the atmosphere to produce chlorine free radicals, which could also catalyse ozone depletion.

As CFCs are chemically inert, their survival time in the atmosphere is relatively long, and they can therefore can find their way into the stratosphere. Here, ultraviolet light can split them into free-radical species, inducing the so-called chlorine catalytic cycle of ozone destruction.

In the United States, this warning led to a ban on the use of CFCs in aerosols. Elsewhere, however, little concern was expressed until a team from the British Antarctic Survey, led by Joseph Farman,

reported in 1985 that severe ozone depletion was occurring over Halley Bay in Antarctica.

The observation was a surprise not just because of its magnitude, but because it was occurring over the South Pole, whereas Rowland and Molina had predicted the largest effects in the tropics, where the intensity of sunlight is greatest.

Unravelling the reasons for polar ozone

\section{IMAGE \\ UNAVAILABLE FOR COPYRIGHT REASONS}

depletion has involved a vast collaborative effort, in which all three recipients have remained active. By the 1990s it had become clear that the low temperatures above Antarctica are critical, and that ozone depletion here is not a result of homogeneous gas-phase chemistry but involves heterogeneous chemical reactions on the
London. The main surprise of this year's Nobel Prize for Physics is not the names of the recipients, but the length of time that has elapsed before their recognition. The prize is to be shared by Martin L. Perl of Stanford University in California for his discovery of the tau lepton in the early 1970s, and Frederick Reines, of the University of California at Irvine, for the detection of the neutrino almost 20 years earlier.

Both discoveries significantly advanced understanding of the subatomic world and helped to establish the so-called Standard Model of particle physics, in which six leptons - consisting of the electron, the muon and the tau and three corresponding neutrinos - and six associated quarks are the elementary constituents of all matter.

The existence of the neutrino was predicted by Wolfgang Pauli in 1933. During the process of 'beta decay', an atomic nucleus emits an electron or positron, and simultaneously changes into the nucleus of a different element. Experiments in the early 1930s showed that energy was irretrievably lost in the process, suggesting that the principle of energy conservation might have to be abandoned for subatomic processes.

To avoid this drastic conclusion, Pauli proposed that the missing energy is carried

surfaces of ice particles in polar stratospheric clouds. These reactions, in the dark of the polar winter, 'prime' the cloud particles for photochemical ozone destruction when sunlight returns in the spring.

In 1987, Molina and his co-workers helped to establish the basis of the chlorine catalytic cycle by highlighting the role of chlorine oxide $(\mathrm{ClO})$ dimers. In collaboration with Neil Harris, Rowland showed that

a the effects of depletion could be seen at all latitudes.

Despite the award's emphasis on ozone, Crutzen's contribution to atmospheric chemistry has been more widespread. "No one has contributed as many ideas to the field," says Cicerone. Crutzen has worked extensively on the chemistry of the troposphere (at altitudes below $10 \mathrm{~km}$ ), studying changes in tropospheric ozone levels (where it is a

pollutant) and identifying the dangers of 'non-industrial pollution' from biomass burning.

Rowland says that atmospheric chemistry was for many years regarded by other chemists as "interesting but not chemistry". Both he and Molina regard the award as a sign that its scientific and social value is now

\section{Particle physics prize arrives at last}

away by an uncharged and massless fundamental particle. It was a radical suggestion, because at than time only three subatomic particles were known - the electron, proton and neutron. Twenty-three years later, Reines and his collaborator Clyde L. Cowan (who died in 1974) announced the detection of the neutrino, calling it "the smallest bit of material reality ever conceived of by man" (see Nature 178, 446-449; 1956).

The tendency of neutrinos to interact with matter is exceedingly weak. To detect them, Reines and Cowan used a 400-litre tank of water and the predicted intense flux of neutrinos available from recently developed nuclear reactors, first in Hanford, Washington State, and later at Savannah River in South Carolina.

Henry Sobel, a colleague of Reines at Irvine, describes the experiment as "a very uncertain proposition, as the conventional wisdom at that time was that these particles were undetectable". But Reines and Cowan reported the detection of a few neutrinos per hour, fully in agreement with theoretical predictions of the time.

Reines has continued an active search for neutrinos. Recently, for example, he helped to direct the project that used an 8,000tonne water Cerenkov detector in a salt 
- mine near Cleveland, Ohio, to search for proton decay. The so-called IMB (Irvine-Michigan-Brookhaven) collaboration shared in the detection of a burst of neutrinos from supernova SN1987A, which strongly corroborated existing theories of the dynamics of stellar collapse.

While Reines

and Cowan knew

what they were

looking for, Perl's

discovery of the tau

lepton was completely unexpected.

"We started out

trying to under-

stand the relation-

ship of the electron

and the muon, but Reines: continuing the we just didn't make search for neutrinos.

any progress," says

Perl. "Finally we started to wonder if maybe there were more leptons; maybe there were a lot of them."

The discovery of the tau was made possible by the construction at the Stanford Linear Accelerator Center (SLAC) of a new accelerator called SPEAR, which Perl and colleagues began using in 1973. In the experiments, electrons and positrons collided at unprecedented energies, giving rise to a plethora of secondary particles.

Painstaking analysis was required to extract the signature of the tau from the raw data. "Our apparatus was primitive and incomplete, and we weren't very good at distinguishing leptons from pions," says Perl. Evidence for the new particle emerged within a couple of years, but Perl says that he and Gary Feldman, now professor of physics at Harvard University, "agonized over the data" before their findings were corroborated by other experiments.

Kurt Gottfried, of Cornell University, admits that when Perl said he had evidence that could only be explained by a new charged lepton, "people were very scepti足 cal”. But he says of Perl and Reines: "These are very
IMAGE

UNAVAILABLE FOR

COPYRIGHT REASONS excellent choices".

Sidney Drell, a colleague of Perl's for more than 30 years at SLAC, describes the award to Perl as a "superb choice"; the experi-

Perl: lepton discoverer ments were "brilliis 'one of a kind'. antly conceived and executed". Perl, he says, is "one of a kind", who likes to experiment off the beaten track.

Perl is currently attempting to detect free quarks. So far, the existence of quarks has been inferred from experiments on composite objects such as the proton. "Some people think this experiment is a waste of time," he says. "But a few have suggested that there may be free quarks left over as relics from the early universe".

Mark Buchanan

\section{French research centres told to make their patents pay}

\& Paris. Elisabeth Dufourcq, France's secretary of state for research, has criticized public research organizations for not doing enough to patent their research results, holding up the aggressive patenting policies of the Atomic Energy Commission (CEA) as an example to follow.

In a bid to encourage researchers to place greater emphasis on patenting, Dufourcq said last week that the government is planning to submit legislation to the National Assembly during its 1995-96 session. This would include giving research teams onequarter of the royalties arising from patents on their work, she said, adding that no ceiling would be imposed on such earnings.

"To submit a patent is to prepare the future, to plant a flag on a new land," said Dufourcq, who was speaking during a visit of the CEA's research centre at Saclay, near Paris. The CEA itself holds almost 1,400 patents, around 900 of which are derived from non-nuclear research. Last year the agency filed 158 patents, and its portfolio earned a substantial income.

In contrast, the patent portfolio of the Centre National de la Recherche Scientifique (CNRS) cost the agency as much as FF125 million in maintenance fees over the past decade. This situation, which critics have attributed in part to CNRS giving too large a share of royalties to licensees, has prompted the agency to review its policy on patenting and to prune back its

\section{Japan accelerates synchrotron plans}

Tokyo. The world's most powerful synchrotron, Japan's SPring-8, is to be commissioned a year ahead of schedule, thanks to an extra $¥ 14.9$ billion (US\$149 million) allocated to the project in a recent special supplementary budget designed to boost the flagging Japanese economy.

Project administrators at the Institute for Physical and Chemical Research (RIKEN), of the Science and Technology Agency (STA), claim that the accelerated schedule has been welcomed by the 900 or so Japanese researchers already recruited into the user's group to carry out experiments on the first 10 beam lines, now due to take place in late 1997. But they admit that the new schedule will make it difficult to recruit the foreign researchers needed to form a large international users' group in time for the commissioning of the $8-\mathrm{GeV}$ synchrotron.

SPring-8 is being built by RIKEN in cooperation with the Japan Atomic Energy Research Institute (JAERI) and the ring will be administered by the Japan Synchro- collection (see Nature 358; 531; 1992).

But the impact of the proposed measures is difficult to predict, particularly because the research organizations already provide individual researchers with a negotiated share of royalties on patents. It is also unclear, for example, whether the government intends to regulate the share of royalties demanded by research organizations from companies licensing their patents.

One senior official from the national biomedical research organization, INSERM, also contests the proposal that there should be no ceiling on researchers' earnings from royalties. Indeed, INSERM has been considering introducing just such a ceiling, he says, as it considers it unfair that some researchers should earn much more than others. The issue of such discrepancies is all the more sensitive, he adds, because INSERM's statutes forbid it to pay bonuses to staff who volunteer for administrative and other duties.

At the Institut Pasteur, whose income from patents tripled between 1988 and 1994, rising from 9 to 18 per cent of its FF860 million budget (see Nature 371, 277; 1994), researchers are not allowed to earn more than FF300,000 annually from royalties. Although the institute is a private organization, it hosts many CNRS and INSERM laboratories, and will need to review its policies when the decree is promulgated, says an official.

Declan Butler

tron Radiation Research Institute (JASRI). User fees have yet to be decided, but a JASRI spokesman says use of the facility's beamlines will essentially be "free".

SPring-8 will eventually incorporate 61 beamlines, in addition to a 1-GeV linac and an 8-GeV synchrotron tunnel with a circumference of $\mathbf{4 0 0}$ metres. The first ten 'public beamlines', whose commissioning has been brought forward to February 1997, will be available for use by researchers from universities and national research institutes not directly associated with RIKEN or JAERI from October 1997.

The beams will be used for a wide variety of studies, including research on protein crystallography, soft X-ray spectroscopy of solids, high-energy inelastic scattering, nuclear resonant scattering, structural analysis of extremely dense states of matter under high pressure, physicochemical analysis, soft X-ray photochemistry, crystal structure analysis, and structural studies of expanded fluids at high temperatures.

Stephen Barker 